\title{
Recent Progress in Flexible Multiferroics
}

\author{
Biswajit Jana ${ }^{1 *}$, Kritika Ghosh ${ }^{1}$, Krishna Rudrapal ${ }^{2}$, Pallavi Gaur ${ }^{1}$, P. K. Shihabudeen ${ }^{1}$ and \\ Ayan Roy Chaudhuri ${ }^{1,2}$
}

${ }^{1}$ Materials Science Centre, Indian Institute of Technology Kharagpur, Kharagpur, India, ${ }^{2}$ Advanced Technology Development Centre, Indian Institute of Technology Kharagpur, Kharagpur, India

A great deal of interest has grown in both academia and industry toward flexible multiferroics in the recent years. The coupling of ferromagnetic properties with ferroelectric properties in multiferroic materials opens up many opportunities in applications such as magnetoelectric random access memories, magnetic field sensors, and energy harvesters. Multiferroic materials on a flexible platform bring an exciting opportunity for the next generation of consumer electronics owing to their unique characteristics of wearability, portability, and weight reduction. However, the fabrication of flexible multiferroic devices is still a great challenge due to various technical difficulties, including the requirement of high growth temperature of the oxide-based multiferroic materials, their lattice mismatch with the flexible substrates, and the brittleness of the functional layers. In this review article, we will discuss different methods of fabricating flexible or even freestanding oxide films to achieve flexible electronics. This article will address the benefits and challenges of each synthesis method in terms of interlayer interactions and growth parameters. Furthermore, the article will include an account of the possible bending limits of different flexible substrates without degrading the properties of the functional layer. Finally, we will address the challenges, opportunities, and future research directions in flexible multiferroics.

Keywords: ferroelectric, ferromagnetic, magnetoelectric coupling, flexible electronics, freestanding oxide films, bending limits

\section{INTRODUCTION}

Ferroic materials are defined by the presence of an order parameter (such as, magnetic, electric, or elastic). Depending on the order parameter, a ferroic material is named ferroelectric (polarization), ferromagnetic (magnetization), ferroelastic (deformation by applied stress), and multiferroic (coexistence of more than one order parameter). It is considered that Hans Schmid first introduced the term "multiferroic" in 1994 [1]. Although the research area of multiferroic is rather recent, multiferroic materials and the possibility of the intrinsic magnetoelectric behavior of solid crystals have been first theoretically predicted by Pierre Curie in 1894 based on symmetry considerations, and the term magnetoelectric coupling was first coined by Debye in 1926 [2]. In 1959, for the first time, Dzyaloshinskii investigated a magnetoelectric effect in $\mathrm{Cr}_{2} \mathrm{O}_{3}$ [3]. For multiferroic materials, the applications of a magnetic or electric field allowing tuning or modifying the electric or magnetic properties, respectively, are considered as magnetoelectric (ME) coupling.

The increasing interest in multiferroics is due to the increased demands toward the development of new multifunctional materials and electronic devices for advanced applications. In recent years, 
numerous materials with diversified structures have been investigated for their multiferroic properties. Also, the ferroic properties of the multiferroic materials prepared in bulk, thin film, composites, heterostructures, or different nanostructures have been reported in the literature. Among different types of materials known for their multiferroic properties, metal oxides with perovskite structure have been widely investigated. For example, magnetic and ferroelectric orderings have been reported in perovskite-type (1-x) $\mathrm{Pb}\left(\mathrm{Fe}_{2 / 3} \mathrm{~W}_{1 / 3}\right) \mathrm{O}_{3-\mathrm{x}} \mathrm{Pb}\left(\mathrm{Mg}_{1 /}\right.$ $\left.{ }_{2} \mathrm{~W}_{1 / 2}\right) \mathrm{O}_{3}$ in early investigations [4-6]. Later, several Pb-based and Bi-based cation ordered and disordered perovskite oxides showing room temperature multiferroic properties have been reported [7-9]. Bi-based perovskite oxides have drawn significant research attention owing to their strong multiferroic behavior. Among the perovskite-type $\mathrm{Bi}$ compounds investigated so far, $\mathrm{BiFeO}_{3}(\mathrm{BFO})$ is a strong room-temperature multiferroic material which in the bulk form possesses antiferromagnetic (Neel temperature $\mathrm{T}_{\mathrm{N}} \sim 643 \mathrm{~K}$ ) and ferroelectric properties with a strong remnant polarization of $\sim 100 \mu \mathrm{C} / \mathrm{cm}^{2}[10,11]$ and Curie temperature $\left(\mathrm{T}_{\mathrm{C}}\right) \sim 1,103 \mathrm{~K}$ [12]. Besides BFO, several other multiferroic materials, such as $\mathrm{BiMnO}_{3}, \mathrm{BiCoO}_{3}$, and $\mathrm{BiCrO}_{3}$, have been reported in the literature [13-16].

Besides the direct coupling of the ferroic orders in bulk multiferroics, elastic strain-mediated magnetoelectric coupling in thin film heterostructures of diverse ferroic materials has been investigated [17]. The ME effect in multiferroic materials is beneficial for designing novel devices such as spintronics device (e.g., MRAM), magnetic field sensors, memory devices, and energy harvestors [18-21]. Furthermore, the ME composites, possessing piezoelectric and magnetostrictive phases, have been investigated for energy harvesting applications, owing to their synergistic effect for both types of energy, that is, mechanical and magnetic. However, in case of thin film multiferroic heterostructures, the functional properties suffer the clamping effect imposed by the rigid substrates which attenuates the coupling of the order parameters. In order to circumvent the substrate clamping effect, the integration of multiferroic thin films and heterostructures onto flexible substrates are being widely explored $[18,22-24]$. In particular, for device application such as the flexible and lightweight energy harvesters on thin flexible polymeric substrate, tiny motion can be captured such as wind, heartbeats, water flows, and respiration movements into electric signals, which can open the door for self-powered flexible electronic devices [25].

Historically, research on designing flexible electronic materials and their heterostructures involves various polymeric substrates, for example, polyimide (PI), polyethylene terephthalate (PET), and polydimethylsiloxane (PDMS), which offer suitable mechanical characteristics for various device applications [26, 27]. However, fabrication of flexible thin films and heterostructures of inorganic ferroic oxides on polymeric flexible substrates involves several process challenges, such as insufficient thermal stability of the substrates at the high growth temperature, required for inorganic oxide layers, their lattice mismatch, and the very fact that the inorganic oxide layers are brittle in nature [28]. In order to circumvent the challenges, various preparation strategies have been developed to combine inorganic multiferroic oxides and the flexible polymeric substrates. The techniques include chemical solution deposition (CSD), transfer process, and laser lift-off (LLO) [22, 29]. Of late, mica has been explored as a viable choice for direct growth of the inorganic functional layers. The van der Waals nature of the bond between the functional oxide layers with mica lifts the complications related to strain originating due to lattice mismatch [28]. Mica, which belongs to the phyllosilicate family, exhibits several key advantages, including chemical inertness, mechanical flexibility, atomically smooth surface, high transparency, and high thermal stability in ultrathin sheets. Commonly, two types of mica substrates are used: fluorophlogopite (F-mica) and muscovite (M-mica). M-mica has a minimum bending radius of $<0.3 \mathrm{~mm}$, when the thickness is $<100 \mathrm{~nm}$. F-mica possesses a bending radius of $<8 \mathrm{~mm}$, when the thickness is approximately $10 \mu \mathrm{m}$ [30]. Furthermore, muscovite and fluorophlogopite have good thermal stability with a temperature up to $600^{\circ} \mathrm{C}$ and $1,000^{\circ} \mathrm{C}$, respectively, which can meet the requirement to grow the functional thin film for most of the inorganic oxide materials [31]. In addition, muscovite and fluorophlogopite single crystal have a layered structure which is advantageous for mechanical exfoliation [31]. Benefiting from the aforementioned features, mica is one of the most potent alternative substrates to prepare flexible inorganic oxide-based multiferroic thin films. van der Waals epitaxy is a popular approach, in which the epitaxial layer of functional oxides can be directly grown on the flexible substrate. The advantages of direct growth with excellent interface quality applying and enhancing the properties of perovskite oxides in flexible memories, flexible sensors, and flexible energy harvesting devices have become an emerging research hot topic.

In this review, we discuss different transfer processes and direct growth approaches that have been utilized for fabricating flexible multiferroics and multiferroic properties on flexible substrates. Finally, promising future development of flexible multiferroics is introduced.

\section{METHODS OF FABRICATING FLEXIBLE MULTIFERROIC FILMS}

To date, flexible multiferroic thin films had been prepared mainly using the laser lift-off (LLO) technique, sacrificial layer-assisted transfer process, and mechanical exfoliation. In such cases, thin films of the chosen multiferroic materials had been deposited on lattice-matched substrates using different growth techniques, for example, sol-gel, pulsed laser deposition (PLD), and magnetron sputtering, which was then lifted by using a laser beam to transfer the multiferroic oxide layer on a flexible substrate [25, 32, 33]. Lee et al. [33] had reported laser lift-off (LLO) of La-doped $\mathrm{Pb}(\mathrm{Zr}, \mathrm{Ti}$ ) $\mathrm{O}_{3}$ and PLZT thin film spin-coated on the sapphire substrate via the sol-gel method which was then transferred to polymeric receptor substrates using a pulsed laser beam. In their work, a sheet of epoxy-based polymer had been laminated as a receptor substrate on top of the functional films. Subsequently, LLO was carried out using a $\mathrm{XeCl}$ pulsed laser beam $(\lambda=308 \mathrm{~nm})$. 
A
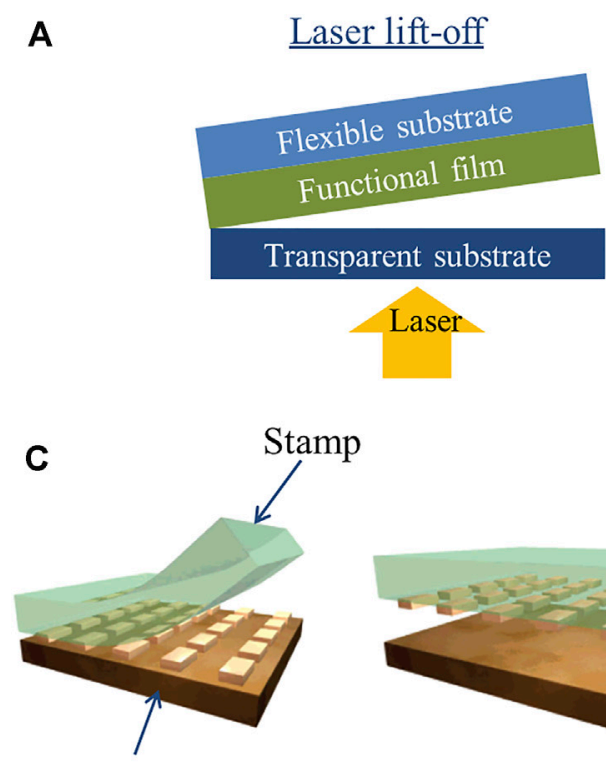

Growth substrate
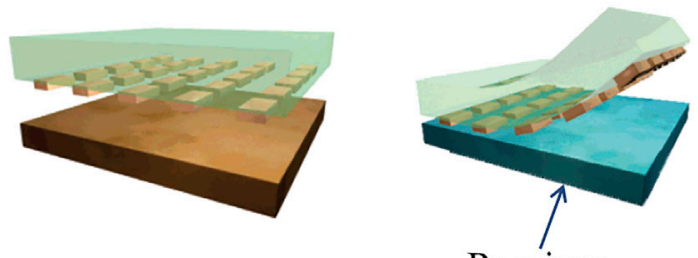

Receiver

substrate

B van der Waals epitaxy
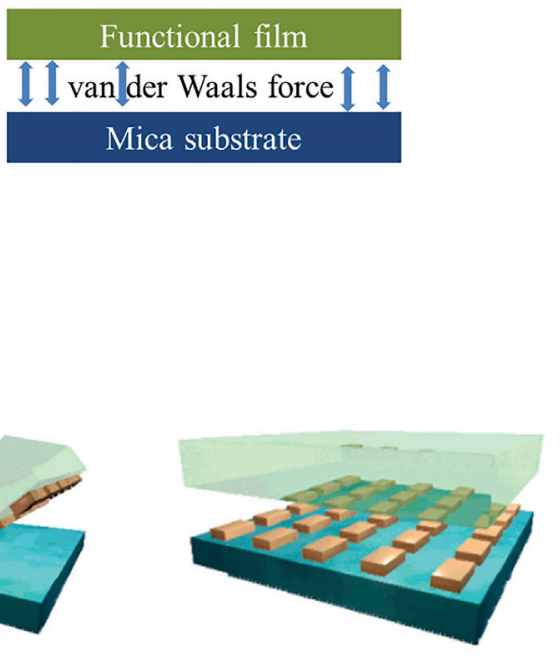

FIGURE 1 | Schematic diagrams of different strategies for high-quality oxide-based multiferroic thin film fabrication. (A) Laser lift-off process, (B) van der Waals epitaxy, and (C) transfer printing. Reprinted with permission from Ref. [42]. Copyright 2007 American Chemical Society.

Figure 1A schematically depicts the LLO process. LLO offers the advantage of fabrication of various high-quality functional layers on compatible substrates, which are transferred to any kind of receiver substrates without incurring serious structural damage or incorporation of impurities [25, 33, 34]. Moreover, LLO lifts the requirement for any high-temperature heat treatment of the receiver substrate.

Another approach that has been employed to prepare flexible multiferroic thin films is the fabrication of the oxide-based functional thin film on a rigid sacrificial substrate, followed by transfer onto flexible substrates by etching the sacrificial layer [35]. The key challenge of this approach is the successful and undamaged separation of the functional thin films from the parent substrates. In this strategy, a sacrificial layer is placed between the functional film and the substrate that is selectively removable by a chemical etchant retaining the properties of the thin films. $\mathrm{SiO}_{2}, \mathrm{MgO}, \mathrm{La}_{0.7} \mathrm{Sr}_{0.3} \mathrm{MnO}_{3}$ (LSMO), and $\mathrm{Sr}_{3} \mathrm{Al}_{2} \mathrm{O}_{6}$ (SAO) have been used as sacrificial layers [33, 36-39] for deposition of multiferroic $\mathrm{BFO}$ and $\mathrm{CoFe}_{2} \mathrm{O}_{4}$ (CFO) thin films on mechanically rigid substrates such as $\mathrm{SrTiO}_{3}$ (STO), $\mathrm{LaAlO}_{3}$ (LAO), $\mathrm{NdGaO}_{3}$, and $\mathrm{TbScO}_{3}$. Zhang et al. [29] prepared freestanding $\mathrm{CFO}$ epitaxial thin films by the transfer process using $\mathrm{MgO}$ as a sacrificial layer, where $\mathrm{CFO}$ epitaxial thin films were grown on MgO-buffered (001) STO substrates by the PLD technique and transferred onto flexible PI substrates. Several steps have been involved in the transfer process. Polystyrene (PS) was first spun on top of the as-grown functional film as a carrier for handling CFO thin films in a subsequent process and then baked at $100^{\circ} \mathrm{C}$ for $15 \mathrm{~min}$ to increase the adhesion between CFO thin film and PS. Then the sacrificial layer was chemically etched in $10 \%\left(\mathrm{NH}_{4}\right)_{2} \mathrm{SO}_{4}$ solution at a temperature of $80^{\circ} \mathrm{C}$. The
$\mathrm{MgO}$ sacrificial layer was etched out completely, after almost $12 \mathrm{~h}$ of chemical etching. Subsequently, the CFO films were kept on the top of the STO substrate through van der Waals adhesion force. After that, the entire specimen was immersed in chloroform to remove the PS layer. Next, by using a waterassisted peel-off technique, the CFO thin film was released onto the surface of water. To mitigate wrinkles and cracks possibly induced by chemical wetting, the CFO thin films remained on the water surface for about a few hours for a slow release. Then the floating film was lifted and transferred onto the flexible PI substrate. Ji et al. [35] fabricated the freestanding BFO film with atomic layer thickness by etching the water-soluble $\mathrm{SAO}$ sacrificial layer. Freestanding BFO films were lifted by dissolving the SAO sacrificial layer in deionized water with mechanical support from PDMS tape or silicon-coated PET and then transferred onto the desired substrates. Furthermore, Zhao et al. [40] prepared freestanding $\mathrm{Fe} / \mathrm{BaTiO}_{3}$ (BTO) multiferroic heterostructures by using the $\mathrm{SAO}$ sacrificial layer. Pesquera et al. [41] reported the fabrication of multiferroic $\mathrm{LSMO} / 0.68 \mathrm{~Pb}\left(\mathrm{Mg}_{1 /}\right.$ $\left.{ }_{3} \mathrm{Nb}_{2 / 3}\right) \mathrm{O}_{3}-0.32 \mathrm{PbTiO}_{3}$ (PMN-PT) heterostructures by the epitaxial transfer technique using $\mathrm{SrRuO}_{3}$ as a sacrificial layer. In their work, an elastomer membrane of polydimethylsiloxane (PDMS) was used to transfer a 45-nm-thick layer of LSMO from its STO (001) growth substrate to platinized PMN-PT (011)pc, after dissolving the intervening epitaxial layer of 30-nm-thick SRO with $\mathrm{NaIO}_{4}(\mathrm{aq})$. The a $\|[100] \mathrm{pc}$ and $\mathrm{b} \|$ [010]pc axes of LSMO that lay parallel to the film edges were approximately aligned with the $\mathrm{x} \|[100] \mathrm{pc}$ and $\mathrm{y} \|[011] \mathrm{pc}$ axes of PMN-PT that lay parallel to the edges of the slightly larger substrate. A schematic diagram for the transfer process is shown in Figure 1C. The advantage of this process is that prior to the 
transferring step, the materials can be doped, deposited, and annealed at high temperature.

However, the aforementioned techniques are not compatible for device fabrication owing to several disadvantages. For example, in the LLO technique, the beam energy of the laser should be lower than that of the band gap of the substrate material and higher than that of the functional layer. These stringent requirements have severely limited the growth of the multiferroic epitaxial layers on specific substrates. These restrictions prevent the opportunity to design and integrate new functional materials for device applications. In addition, the multiferroic thin films can also be damaged by the etchant used in the sacrificial layer-assisted transfer process. To circumvent those problems, direct growth techniques have been proved to be a viable route to grow the multiferroic thin films directly on flexible substrates.

The solution-based process is a popular direct growth method to fabricate multiferroic thin films on flexible polymeric substrates due to their wide range of advantages such as largearea deposition, easy control of composition, atmospheric processing, and low equipment cost [22]. Among various solution-based processes, chemical solution deposition (CSD) has been widely employed to prepare crystalline functional thin films. For the first time, Tomczyk et al. [22] fabricated multiferroic spin-coated BFO thin films directly on the flexible polyimide substrates at a temperature as low as $300^{\circ} \mathrm{C}$ by using the CSD-based seeded photosensitive precursor method. In their work, the proposed synthesis technique consisted of several steps. First, photoactive sol was prepared. Second, the prepared $\mathrm{BFO}$ seeds were incorporated into the photoactive sol, which increased the number of nucleation sites in the film, helping a further reduction of the crystallization temperature. Third, the deposition of seeded photoactive sol on the polyimide substrates and irradiation by a high-intensity UV excimer lamp took place. Last, the irradiated layer was heattreated by rapid thermal processing. The CSD-based method can be used for the potential integration of multiferroic BFO thin films with flexible polymeric substrates and may have a wide impact in terms of the extended use of functional oxide thin films fabricated with a low thermal budget. However, many challenges exist for CSD prepared BFO thin films such as unsaturated or leaky P-E loops at room temperature [43] and small polarization value or a large coercive field [22].

An improved direct growth technique for minimizing the substrate constraint was demonstrated by several research groups by fabricating multiferroic thin films on a flexible mica substrate via van der Waals epitaxy [23, 44, 45]. As mentioned earlier, mica is well suited to overcome the problems associated with the polymeric substrates. van der Waals (vdW) epitaxy is defined as the growth of a layered material on another layered substrate (Figure 1B). In vdW epitaxy, the substrate surface is free from any dangling bond that allows the formation of interface between the surface and the overgrown layer with less number of defects. Thus, vdW heteroepitaxy on muscovite provides an excellent platform to develop flexible devices based on oxide heteroepitaxy without strain and substrate clamping. Sun et al. [46] fabricated Au/ $\mathrm{BFO} / \mathrm{SRO} / \mathrm{BTO} / \mathrm{F}-\mathrm{mica}$ heterostructures using the RF magnetron sputtering technique. Amrillah et al. [45] deposited self-assembled multiferroic BFO-CFO heterojunction on a mica substrate via the PLD technique where the substrate was heated to $650^{\circ} \mathrm{C}$. A CFO interfacial buffer layer was used and deposited prior to the deposition of the BFO-CFO heterojunction on mica. This demonstrates that the fabrication of multiferroic thin films can be achieved on mica substrates without resorting to substrate-induced epitaxial strain. The vdW epitaxy not requiring lattice matching conditions does have the advantage of reducing the possibility of structural defects created at the interface to propagate across the film [44, 45]. In vdW epitaxy, the epitaxial layer can be grown with a lattice mismatch of $60 \%$ between the functional layer and the substrate [47]. Hence, as an alternative strategy, the prospects of vdW epitaxy are quite attractive toward the successful growth of multiferroic thin films on a flexible platform.

\section{MULTIFERROIC PROPERTIES ON FLEXIBLE SUBSTRATES}

In the process of development of flexible multiferroics, the coupling between ferroic states and mechanical deformation should be of concern, including magnetostrictive properties, piezoelectric properties, ferroelectric properties, and ferromagnetic properties. Tomczyk et al. [22] investigated the multiferroic behavior of CSD-based BFO thin films on the flexible polyimide substrate (bending radii, $\mathrm{R} \sim 20 \mathrm{~mm}$ ) which demonstrated a remanent polarization of $2.8 \mu \mathrm{C} / \mathrm{cm}^{2}$ with a coercive field of $380 \mathrm{kV} / \mathrm{cm}$. In addition, the BFO films exhibited a ferromagnetic response at room temperature. Liu et al. [48] demonstrated promising magnetostriction properties of the $\mathrm{CoFe}_{2} \mathrm{O}_{4}$ (CFO) epitaxial thin films deposited on muscovite mica. The reported saturation magnetization (Ms) and magnetostriction coefficient $(\triangle \lambda)$ of the flexible CFO thin film is $120-150 \mathrm{emu} / \mathrm{cm}^{3}$ and $-104 \mathrm{ppm}$, respectively. From the results of this work, it was observed that the hysteresis loops remained unaltered under different bending conditions. Furthermore, Feng et al. [49] predicted that the magnetoelectrical properties strongly improved in the case of LSMO/BFO superlattice. Zhao et al. [40] demonstrated the growth of multiferroic $\mathrm{Fe} / \mathrm{BaTiO}_{3}$ (BTO) heterostructures by using the PLD technique, followed by transfer of the Fe/BTO functional layer on PDMS substrates. The flexible Fe/BTO/PDMS heterostructures exhibited good ferroelectric and ferromagnetic behavior with a bending radius of $5 \mathrm{~mm}$. Sun et al. [46] successfully fabricated high-quality flexible Au/BFO/SRO/BTO/ mica heterostructures using the magnetron sputtering technique. SRO/BTO double buffer layers lower the lattice mismatch-induced strain on BFO and enhance the quality of the epitaxial heterostructures. The prepared BFO-based heterostructures showed the largest polarization $\left(\mathrm{P}_{\mathrm{s}}=100 \mu \mathrm{C} /\right.$ $\mathrm{cm}^{2}, \mathrm{P}_{\mathrm{r}} \sim 97 \mu \mathrm{C} / \mathrm{cm}^{2}$ ), and it was very stable after $10^{4}$ bending cycles under a $5-\mathrm{mm}$ bending radius. This study demonstrated the feasibility of fabricating high-quality BFO films on flexible mica substrates with excellent ferroelectric properties. However, 

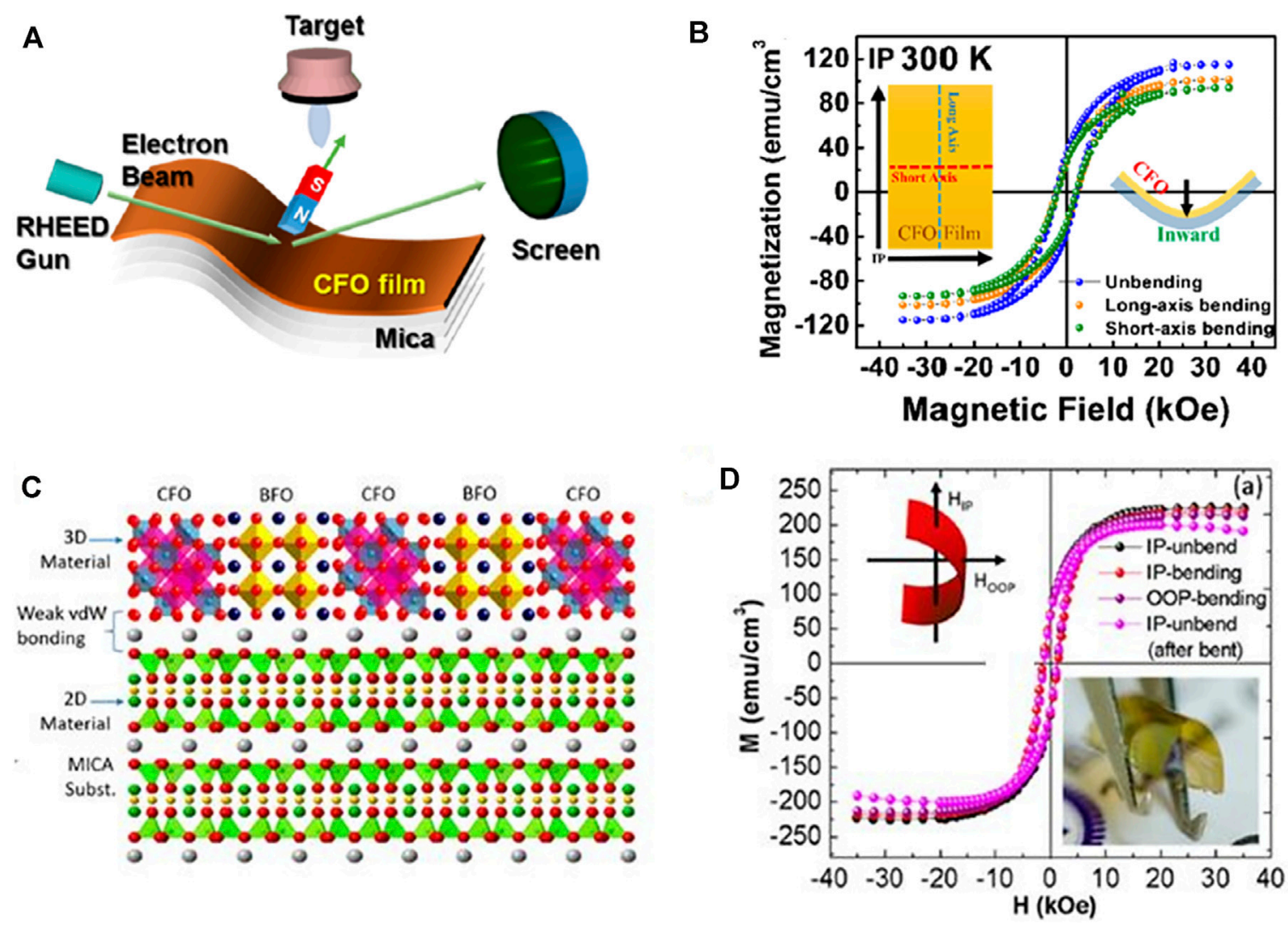

FIGURE 2 | (A) Schematic of deposition process for flexible CFO film monitored by RHEED in real time; (B) the magnetic hysteresis loops show that the flexible CFO/mica exhibits solid magnetic properties regardless of bending. Reprinted with permission from Ref. [48]. Copyright 2017 American Chemical Society. (C) Crystal structure of the interface region between the CFO-BFO composite and mica substrate; (D) the M-H loops of BFO-CFO under bending. Reprinted with permission from Ref. [45]. Copyright 2017 American Chemical Society.

the magnetoelectric coupling strength of the prepared BFO thin film heterostructure was not revealed.

The clamping effect induced by the rigid substrate has always been a key factor to weaken the ME coupling. To overcome this problem, Amrillah et al. [45] synthesized van der Waals epitaxy self-assembled BFO-CFO bulk heterojunctions on M-mica. The reason behind this approach was that the bulk heterojunction interacts with the substrate via weak van der Waals forces (Figure 2C); the interface was incoherent, and this greatly mitigated the clamping effect. The ME coupling coefficient $\left(a_{\mathrm{ME}}\right)$ of the BFO-CFO bulk heterojunctions was attainable up to $74 \mathrm{mV} /(\mathrm{cm} . \mathrm{Oe})$. Furthermore, it was observed that the out-ofplane (OOP) converse piezoelectric coefficient $\left(\mathrm{d}_{33}\right)$ value of the BFO-CFO bulk heterojunction on mica was $76.5 \mathrm{pm} \mathrm{V}^{-1}$, higher than that on the rigid STO (111) substrate $\left(50 \mathrm{pm} \mathrm{V}^{-1}\right)$ grown under similar conditions. This reported value is also higher than $\mathrm{d}_{33}$ in (100)-, (110)-, and (111)-oriented BFO-CFO/STO heterostructures (50, 40, and $30 \mathrm{pm} \mathrm{V}^{-1}$, respectively) [50]. The reported results suggested that the improved piezoelectric response of BFO pillars embedded in the CFO matrix derived from the release of the substrate constraint. It is substantial to mention that $\mathrm{BFO}-\mathrm{CFO}$ heterostructure on a flexible mica substrate exhibited superior magnetoelectric properties as compared to that on the rigid substrate. Figure 2D shows the $\mathrm{M}-\mathrm{H}$ loop under different bending modes. The magnetic hysteresis loop remained the same even after bending at a bending radius of $2 \mathrm{~mm}$, indicating that the ME coupling of this system is resilient against mechanical bending, which is necessary for the development of the flexible ME heterostructure thin films in flexible and wearable devices.

\section{SUMMARY AND FUTURE PERSPECTIVES}

Arriving at the conclusion of this brief review, it has been observed that remarkable progress has been made in the last few years of this emerging hot topic of research. A significant progress has been achieved in terms of growth techniques and the properties of multiferroic thin films on the flexible platforms. It is believed that flexible multiferroic materials have a promising future in various electronic device applications because of their rich multifunctional properties and the research achievements which have been gained by different research groups so far. By achieving the flexibility of oxides multiferroic films, they can show great potential in wearable devices. Currently, the main strategies to the flexibility of high-quality functional oxide films are LLO and transferring films to flexible substrates via etching 
the sacrificial layer. Nevertheless, as it has been discussed in this article, there are some limitations and problems in the fabrication of stable and high-quality flexible multiferroic films through transfer processes. Recently, van der Waals epitaxy has been proven to be an alternative route to fabricate the high-quality flexible multiferroic films. Still, there is plenty of space left to be improved and investigated for the flexible multiferroic research domain. The development of multiferroic materials, methods, and properties and recent emergence of flexible devices motivate research communities to look forward to the application of multiferroic-based flexible devices.

\section{REFERENCES}

1. Schmid H Multi-ferroic Magnetoelectrics. Ferroelectrics (1994) 162:317-38. doi:10.1080/00150199408245120

2. Fiebig M Revival of the Magnetoelectric Effect. J Phys D: Appl Phys (2005) 38: R123-52. doi:10.1088/0022-3727/38/8/r01

3. Dzyaloshinskii IE On the Magneto-Electrical Effects in Antiferromagnets. Sov Phys JETP (1960) 10:628-9.

4. Cross LE Ferroic Materials and Composites: Past, Present and Future. Adv Ceramics 1(1990) 71-102. doi:10.1007/978-94-009-0763-8_5

5. Spaldin NA, Fiebig M Materials Science. The Renaissance of Magnetoelectric Multiferroics. Science (2005) 309:391-2. doi:10.1126/science.1113357

6. Ascher E, Rieder H, Schmid H, Stössel H Some Properties of Ferromagnetoelectric Nickel-Iodine Boracite, Ni3B7O13I. I Appl Phys (1966) 37:1404-5. doi:10.1063/1.1708493

7. Lehmann AG, Sciau P Ferroelastic Symmetry Changes in the Perovskite PbFeY0.5Ta0.5O3. J Phys Condens Matter (1999) 11:1235-45. doi:10.1088/ 0953-8984/11/5/011

8. Lampis N, Sciau P, Lehmann AG Rietveld Refinements of the Paraelectric and Ferroelectric Structures of PbFe0.5Ta0.5O3. J Phys Condens Matter (2000) 12: 2367-78. doi:10.1088/0953-8984/12/11/303

9. Kumar A, Rivera I, Katiyar RS, Scott JF Multiferroic $\mathrm{Pb}(\mathrm{Fe} 0.66 \mathrm{~W} 0.33)$ 0.80Ti0.20O3 Thin Films: A Room-Temperature Relaxor Ferroelectric and Weak Ferromagnetic. Appl Phys Lett (2008) 92:132913. doi:10.1063/1.2906371

10. Sando D, Barthélémy A, Bibes M BiFeO3epitaxial Thin Films and Devices: Past, Present and Future. J Phys Condens Matter (2014) 26:473201. doi:10.1088/0953-8984/26/47/473201

11. Yun KY, Ricinschi D, Kanashima T, Noda M, Okuyama M Giant Ferroelectric Polarization beyond $150 \mu \mathrm{C} / \mathrm{cm} 2$ in BiFeO3Thin Film. Jpn J Appl Phys (2004) 43:L647-L648. doi:10.1143/jjap.43.1647

12. Zhang Q, Sando D, Nagarajan V Chemical Route Derived Bismuth Ferrite Thin Films and Nanomaterials. J Mater Chem C (2016) 4:4092-124. doi:10.1039/c6tc00243a

13. Amrillah T, Quynh LT, Nguyen Van C, Do TH, Arenholz E, Juang J-Y, et al. Flexible Epsilon Iron Oxide Thin Films. ACS Appl Mater Inter (2021) 13: 17006-12. doi:10.1021/acsami.0c23104

14. Banerjee S, Tyagi AK Functional Materials: Preparation, Processing and Applications (2011).

15. Menéndez C, Chu D, Cazorla C Oxygen-vacancy Induced Magnetic Phase Transitions in Multiferroic Thin Films. Npj Comput Mater (2020) 6:1-11. doi:10.1038/s41524-020-0344-3

16. Nechache R, Harnagea C, Pignolet A Multiferroic Properties-Structure Relationships in Epitaxial $\mathrm{Bi}(2) \mathrm{FeCrO}(6)$ Thin Films: Recent Developments. J Phys Condens Matter (2012) 24:096001. doi:10.1088/0953-8984/24/9/096001

17. Sun F, Chen D, Gao X, Liu J-M Emergent Strain Engineering of Multiferroic BiFeO3 Thin Films. J Materiomics (2021) 7:281-94. doi:10.1016/ j.jmat.2020.08.005

18. Jing WQ, Fang F A Flexible Multiferroic Composite with High Self-Biased Magnetoelectric Coupling. Composites Sci Technology (2017) 153:145-50. doi:10.1016/j.compscitech.2017.10.010

\section{AUTHOR CONTRIBUTIONS}

All authors made a substantial, direct, and intellectual contribution to the work and approved it for publication.

\section{ACKNOWLEDGMENTS}

We acknowledge the Ministry of Education, India (SPARC/20182019/P252/SL dated 15-03-2019). KG wishes to acknowledge the financial support of DST-INSPIRE fellowship (no. IF180046).

19. Vopson MM Fundamentals of Multiferroic Materials and Their Possible Applications. Crit Rev Solid State Mater Sci (2015) 40:223-50. doi:10.1080/ 10408436.2014.992584

20. Panda D, Tseng T-Y Growth, Dielectric Properties, and Memory Device Applications of ZrO2 Thin Films. Thin Solid Films (2013) 531:1-20. doi:10.1016/j.tsf.2013.01.004

21. Ma J, Hu J, Li Z, Nan C-W Recent Progress in Multiferroic Magnetoelectric Composites: from Bulk to Thin Films. Adv Mater 23 (2011). p. 1062-87. doi:10.1002/adma.201003636

22. Tomczyk M, Bretos I, Jiménez R, Mahajan A, Ramana EV, Calzada ML, et al. Direct Fabrication of BiFeO3 Thin Films on Polyimide Substrates for Flexible Electronics. J Mater Chem C (2017) 5:12529-37. doi:10.1039/c7tc04571a

23. Yang C, Lv P, Qian J, Han Y, Ouyang J, Lin X, et al. Fatigue-Free and BendingEndurable Flexible Mn-Doped $\mathrm{Na} 0.5 \mathrm{Bi} 0.5 \mathrm{TiO} 3$-BaTiO 3 -BiFeO 3 Film Capacitor with an Ultrahigh Energy Storage Performance. Adv Energ Mater. (2019) 9:1803949-10. doi:10.1002/aenm.201803949

24. Liu W, Wang H Flexible Oxide Epitaxial Thin Films for Wearable Electronics: Fabrication, Physical Properties, and Applications. J Materiomics (2020) 6: 385-96. doi:10.1016/j.jmat.2019.12.006

25. Park K-I, Son JH, Hwang G-T, Jeong CK, Ryu J, Koo M, et al. Highly-efficient, Flexible Piezoelectric PZT Thin Film Nanogenerator on Plastic Substrates. Adv Mater (2014) 26:2514-20. doi:10.1002/adma.201305659

26. Nathan A, Ahnood A, Cole MT, Sungsik Lee S, Suzuki Y, Hiralal P, et al. Flexible Electronics: The Next Ubiquitous Platform. Proc IEEE (2012) 100: 1486-517. doi:10.1109/JPROC.2012.2190168

27. Malik A, Kandasubramanian B Flexible Polymeric Substrates for Electronic Applications. Polym Rev (2018) 58:630-67. doi:10.1080/ 15583724.2018.1473424

28. Zhong G, Li J Muscovite Mica as a Universal Platform for Flexible Electronics. J Materiomics (2020) 6:455-7. doi:10.1016/j.jmat.2019.12.004

29. Zhang Y, Shen L, Liu M, Li X, Lu X, Lu L, et al. Flexible Quasi-TwoDimensional CoFe2O4 Epitaxial Thin Films for Continuous Strain Tuning of Magnetic Properties. ACS Nano, 11 (2017). p. 8002-9. doi:10.1021/ acsnano.7b02637

30. He Y, Dong H, Meng Q, Jiang L, Shao W, He L, et al. Mica, a Potential TwoDimensional-Crystal Gate Insulator for Organic Field-Effect Transistors. Adv Mater (2011) 23:5502-7. doi:10.1002/adma.201103592

31. Liu W, Wang H Flexible Oxide Epitaxial Thin Films for Wearable Electronics: Fabrication, Physical Properties, and Applications. J Materiomics (2020) 6(2): 385-96. doi:10.1016/j.jmat.2019.12.006

32. Zhang Y, Ma C, Lu X, Liu M Recent Progress on Flexible Inorganic SingleCrystalline Functional Oxide Films for Advanced Electronics. Mater Horiz (2019) 6:911-30. doi:10.1039/c8mh01598h

33. Lee CH, Kim SJ, Oh Y, Kim MY, Yoon Y-J, Lee H-S Use of Laser Lift-Off for Flexible Device Applications. J Appl Phys (2010) 108:102814-5. doi:10.1063/ 1.3511716

34. You A, Be MAY Damage-free Separation of GaN Thin Films from Sapphire Substrates. Appl Phys Lett (2004) 72(5):599-601. doi:10.1063/1.120816

35. Ji D, Cai S, Paudel TR, Sun H, Zhang C, Han L, et al. Freestanding Crystalline Oxide Perovskites Down to the Monolayer Limit. Nature (2019) 570:87-90. doi:10.1038/s41586-019-1255-7 
36. Le PTP, ten Elshof JE, Koster G Epitaxial Lift-Off of Freestanding (011) and (111) SrRuO3 Thin Films Using a Water Sacrificial Layer. Sci Rep (2021) 11: 1-8. doi:10.1038/s41598-021-91848-2

37. Lu D, Baek DJ, Hong SS, Kourkoutis LF, Hikita Y, Hwang HY Synthesis of Freestanding Single-crystal Perovskite Films and Heterostructures by Etching of Sacrificial Water-Soluble Layers. Nat Mater (2016) 15:1255-60. doi:10.1038/ nmat 4749

38. Dagdeviren C, Su Y, Joe P, Yona R, Liu Y, Kim Y-S, et al. Conformable Amplified lead Zirconate Titanate Sensors with Enhanced Piezoelectric Response for Cutaneous Pressure Monitoring. Nat Commun (2014) 5:5. doi:10.1038/ncomms5496

39. Dagdeviren C, Shi Y, Joe P, Ghaffari R, Balooch G, Usgaonkar K, et al. Conformal Piezoelectric Systems for Clinical and Experimental Characterization of Soft Tissue Biomechanics. Nat Mater (2015) 14:728-36. doi:10.1038/nmat4289

40. Zhao Y, Peng R, Guo Y, Liu Z, Dong Y, Zhao S, et al. Ultraflexible and Malleable $\mathrm{Fe} / \mathrm{BaTiO} 3$ Multiferroic Heterostructures for Functional Devices. Adv Funct Mater (2021) 31:2009376-6. doi:10.1002/ adfm.202009376

41. Pesquera D, Khestanova E, Ghidini M, Zhang S, Rooney AP, Maccherozzi F, et al. Large Magnetoelectric Coupling in Multiferroic Oxide Heterostructures Assembled via Epitaxial Lift-Off. Nat Commun (2020) 11:1-8. doi:10.1038/ s41467-020-16942-x

42. Feng X, Meitl MA, Bowen AM, Huang Y, Nuzzo RG, Rogers JA Competing Fracture in Kinetically Controlled Transfer Printing. Langmuir (2007) 23: 12555-60. doi:10.1021/la701555n

43. Yang B, Jin L, Wei R, Tang X, Hu L, Tong P, et al. Chemical Solution Route for High-Quality Multiferroic BiFeO 3 Thin Films. Small (2021) 17:1903663. doi:10.1002/smll.201903663

44. Bitla Y, Chu Y-H MICAtronics: A New Platform for Flexible X-Tronics. FlatChem (2017) 3:26-42. doi:10.1016/j.flatc.2017.06.003

45. Amrillah T, Bitla Y, Shin K, Yang T, Hsieh Y-H, Chiou Y-Y, et al. Flexible Multiferroic Bulk Heterojunction with Giant Magnetoelectric Coupling via van der Waals Epitaxy. ACS Nano (2017) 11:6122-30. doi:10.1021/ acsnano.7b02102
46. Sun H, Luo Z, Zhao L, Liu C, Ma C, Lin Y, et al. BiFeO3-Based Flexible Ferroelectric Memristors for Neuromorphic Pattern Recognition. ACS Appl Electron Mater (2020) 2:1081-9. doi:10.1021/acsaelm.0c00094

47. Utama MIB, De La Mata M, Magen C, Arbiol J, Xiong Q Twinning-, polytypism-, and polarity-induced morphological modulation in nonplanar nanostructures with van der Waals Epitaxy. Adv Funct Mater (2013) 23: 1636-46. doi:10.1002/adfm.201202027

48. Liu H-J, Wang C-K, Su D, Amrillah T, Hsieh Y-H, Wu K-H, et al. Flexible Heteroepitaxy of CoFe2O4/muscovite Bimorph with Large Magnetostriction. ACS Appl Mater Inter (2017) 9:7297-304. doi:10.1021/acsami.6b16485

49. Feng N, Mi W, Wang X, Cheng Y, Schwingenschlögl U Superior Properties of Energetically Stable La2/3Sr1/3MnO3/tetragonal $\mathrm{BiFeO} 3$ Multiferroic Superlattices. ACS Appl Mater Inter (2015) 7:10612-6. doi:10.1021/ acsami.5b02436

50. Yan L, Wang Z, Xing Z, Li J, Viehland D Magnetoelectric and Multiferroic Properties of Variously Oriented Epitaxial BiFeO3-CoFe2O4 Nanostructured Thin Films. J Appl Phys, 107 (2010). 0641061-6. doi:10.1063/1.3359650

Conflict of Interest: The authors declare that the research was conducted in the absence of any commercial or financial relationships that could be construed as a potential conflict of interest.

Publisher's Note: All claims expressed in this article are solely those of the authors and do not necessarily represent those of their affiliated organizations, or those of the publisher, the editors, and the reviewers. Any product that may be evaluated in this article, or claim that may be made by its manufacturer, is not guaranteed or endorsed by the publisher.

Copyright $\odot 2022$ Jana, Ghosh, Rudrapal, Gaur, Shihabudeen and Roy Chaudhuri. This is an open-access article distributed under the terms of the Creative Commons Attribution License (CC BY). The use, distribution or reproduction in other forums is permitted, provided the original author(s) and the copyright owner(s) are credited and that the original publication in this journal is cited, in accordance with accepted academic practice. No use, distribution or reproduction is permitted which does not comply with these terms. 